\title{
Cervical Length and the Risk of Microbial Invasion of the Amniotic Cavity in Women with Preterm Premature Rupture of Membranes
}

\begin{abstract}
The aims of this study were to determine whether sonographically measured cervical length is of value in the identification of microbial invasion of the amniotic cavity in women with preterm premature rupture of membranes (PPROM) and to compare its performance with maternal blood C-reactive protein (CRP), white blood cell count (WBC), and amniotic fluid (AF) WBC. This prospective observational study enrolled 50 singleton pregnancies with PPROM. Transvaginal ultrasound for measurement of cervical length was performed and maternal blood was collected for the determination of CRP and WBC at the time of amniocentesis. AF obtained by amniocentesis was cultured and WBC determined. The prevalence of a positive amniotic fluid culture was $26 \%$ (13/50). Patients with positive amniotic fluid cultures had a significantly shorter median cervical length and higher median CRP, WBC, and AF WBC than did those with negative cultures. Multiple logistic regression indicated that only cervical length had a significant relationship with the log odds of a positive AF culture. Transvaginal sonographic measurement of cervical length is valuable in the identification of microbial invasion of amniotic cavity in women with PPROM. Cervical length performs better than AF WBC, maternal blood CRP, and WBC in the identification of a positive amniotic fluid culture.
\end{abstract}

Key Words : Cervical Length; Microbial Invasion of the Amniotic Cavity; Preterm Premature Rupture of Membranes

\author{
Joon-Seok Hong, Kyo Hoon Park, \\ Jae-Hong Noh, Young-Hoon Suh \\ Department of Obstetrics and Gynecology, Seoul \\ National University College of Medicine, Seoul, Korea
}

Received : 27 September 2006

Accepted : 28 December 2006

Address for correspondence

Kyo Hoon Park, M.D.

Department of Obstetrics and Gynecology, Seoul

National University Bundang Hospital, 300

Gumi-dong, Bundang-gu Seongnam 463-707, Korea

Tel : +82.31-787-7252, Fax : +82.31-787-4054

E-mail : pkh0419@snubh.org

\section{INTRODUCTION}

Preterm premature rupture of membranes (PPROM) is a leading cause of perinatal morbidity and mortality (1). Moreover, microbial invasion of the amniotic cavity is present in about 30\% of patients with PPROM (2-5), and is a risk factor of impending spontaneous delivery and of an adverse perinatal outcome (4-6). Antenatal diagnosis of microbial invasion of the amniotic cavity requires amniocentesis and amniotic fluid analysis, but amniocentesis is an invasive method and may not be available in all clinics. Moreover, amniocentesis may not be feasible when the volume of amniotic fluid is reduced in PPROM patients, and culture results may not be available for several days, and thus not available for immediate management decisions. Therefore, alternative, non-invasive approaches are required to identify pregnancies at risk of microbial invasion of the amniotic cavity $(7,8)$

Transvaginal ultrasound of the cervix is a well recognized, noninvasive, objective tool for the evaluation of cervical status. Moreover, cervical length as determined by transvaginal ultrasound has emerged as a powerful means of predicting spontaneous preterm delivery in both women with preterm labor and intact membranes, and in women with PPROM (9-13). Recently, it was suggested that a short cervix in women with preterm labor and intact membranes is a risk factor for microbial invasion of the amniotic cavity $(14,15)$. Therefore, we considered that if this is the case in women with preterm labor and intact membranes, it is possible that women with PPROM and a shorter cervix may also be at greater risk of microbial invasion of amniotic cavity. Thus, the purposes of this study were to determine whether sonographically determined cervical length is of value in terms of identifying microbial invasion of the amniotic cavity in women with PP$\mathrm{ROM}$, and to compare its merits versus maternal blood Creactive protein (CRP), white blood cell (WBC) count, and amniotic fluid (AF) WBC.

\section{MATERIALS AND METHODS}

\section{Study design}

The study population consisted of consecutive patients who were admitted to Seoul National University Bundang Hospital with the diagnosis of PPROM ( $<36$ weeks of gestation) and singleton gestation who underwent cervical measurement by ultrasound and amniocentesis for assessment of microbiologic status of the amniotic cavity and fetal lung maturity 
between May 2003 and May 2006. At our institution, cervical length measurement and amniocentesis for retrieval of amniotic fluid are offered routinely to all patients who are admitted with the diagnosis of PPROM. We usually performed amniocentesis before the initiation of antibiotic treatments. Rupture of membranes was diagnosed by examination with a sterile speculum and a combination of vaginal pooling of $\mathrm{AF}$ and nitrazine and ferning tests. The presence of microbial invasion of the amniotic cavity was defined as a positive amniotic fluid culture. Written informed consent was obtained from all subjects to donate AF for research purposes. The institutional review board of Seoul National University Bundang Hospital approved the collection and utilization of the biological materials and clinical data for research purposes.

\section{Amniotic fluid}

Amniotic fluid was retrieved by transabdominal amniocentesis and cultured for aerobic and anaerobic bacteria and for Mycoplasmas (Ureaplasma urealyticum and Mycoplasma hominis) according to the methods previously described (16). An amniotic fluid WBC count was determined with a hemocytometer chamber.

\section{Maternal blood}

Maternal blood was drawn for the determination of WBC and $\mathrm{C}$-reactive protein concentration at amniocentesis. Maternal blood WBC was determined using a flow cytometry (ADVIA 120, Bayer, Long Island, NY, U.S.A.). C-reactive protein quantitation was performed by particle enhanced turbidimetric immunoassay using Dimension RxL Autoanalyzer (DADE Behring, Deerfield, IL, U.S.A.). The detection linearity ranged from $0.05-25 \mathrm{mg} / \mathrm{dL}$.

\section{Transvaginal ultrasound examination of the cervix}

At amniocentesis, transvaginal ultrasonographic assessment of cervical length were performed using an Envisor (Philips Medical System, Eindhorven, Netherlands) ultrasound machine with a $6.0 \mathrm{MHz}$ transducer. The women were asked to empty their bladder and were placed in the dorsal lithotomy position. The probe was gently placed at the anterior fornix of the vagina to obtain a sagittal view of the complete cervix, including the internal os, external os, and endocervical canal. The probe was slowly withdrawn until the image blurred and then the insertion pressure was increased only enough to restore a clear cervical image. This cervical image was magnified to at least $75 \%$ of the screen, and the electronic markers were placed at the furthest points between the internal os and external os, then the cervical length was measured as a straight line. The shortest of three measurements obtained was taken as the cervical length.

\section{Statistical analysis}

Univariate analysis was conducted using Mann-Whitney $\mathrm{U}$ test or Fisher's exact test as appropriate. A receiver operating characteristic (ROC) curve was used to describe the relationship between the sensitivity (true-positive rate) and the false-positive rate for different values of cervical length in the identification of microbial invasion of the amniotic cavity. Multiple logistic regression analysis was used to examine the relationship between cervical length and microbial invasion of the amniotic cavity, controlling for the effect of potential compounding variables.

\section{RESULTS}

Fifty patients met the entry criteria in this study. The mean gestational age at amniocentesis was 31.4 weeks $( \pm 3.2)$, while mean gestational age at delivery was 33.7 weeks ( \pm 3.1). The median amniocentesis-to-delivery interval was 101 $\mathrm{hr}$ (range 1-2,477 hr), and the mean time interval from rupture of membranes to amniocentesis was $23.4 \mathrm{hr}$ ( \pm 34.7 ). The prevalence of positive amniotic fluid culture was $26 \%$ (13/50). Microorganisms isolated from the amniotic fluid were Ureaplasma urealyticum $(\mathrm{n}=10)$, Streptococcus agalactiae $(\mathrm{n}=1), G(-) \operatorname{rod}(\mathrm{n}=1), G(+) \operatorname{cocci}(\mathrm{n}=1)$.

Table 1 describes the clinical characteristics and outcomes of the study population according to the results of amniotic fluid culture. There were no significant differences in the mean maternal age, median gestational age at amniocentesis, and median time interval from membrane rupture to amniocentesis between the two groups. However, the patients

Table 1. Clinical characteristics and outcomes of study population according to the amniotic fluid culture results

\begin{tabular}{cccc}
\hline & AF culture (-) & AF culture (+) & $p$ \\
& $\mathrm{~N}=37$ & $\mathrm{~N}=13$ & value \\
\hline Maternal age (yr, mean $\pm \mathrm{SD})$ & $31.0 \pm 3.4$ & $30.6 \pm 4.4$ & $\mathrm{NS}$ \\
Gestational age at amniocentesis & 32.3 & 31.4 & $\mathrm{NS}$ \\
(weeks, median and range) & $(21.3-35.4)$ & $(27.2-35.0)$ & \\
Gestational age at delivery & 34.2 & 32.2 & $<0.001$ \\
(weeks, median and range) & $(25.3-41.3)$ & $(28.0-35.1)$ & \\
Cervical length (cm, median & 3.3 & 2.3 & 0.037 \\
and range) & $(0.83-7.89)$ & $(0.0-4.24)$ & \\
Maternal CRP (mg/dL, & 0.35 & 0.65 & 0.004 \\
median and range) & $(0.01-7.25)$ & $(0.15-6.29)$ & \\
Maternal WBC (cells/ $\mu \mathrm{L}$, & 8,820 & 12,710 & 0.025 \\
median and range) & $(6,140-21,560)$ & $(4,740-23,150)$ \\
AF WBC (cells/ $\mu \mathrm{L}$, & 5.0 & 172 & $<0.001$ \\
median and range) & $(0-60)$ & $(1-11,000)$ & \\
Interval from membrane & 10 & 11 & $\mathrm{NS}$ \\
rupture to amniocentesis & $(2-165)$ & $(5-39)$ & \\
(hr, median and range) & \multicolumn{3}{c}{} \\
\hline
\end{tabular}

AF, amniotic fluid; NS, not significant; SD, standard deviation; CRP, Creactive protein; WBC, white blood cell count. 


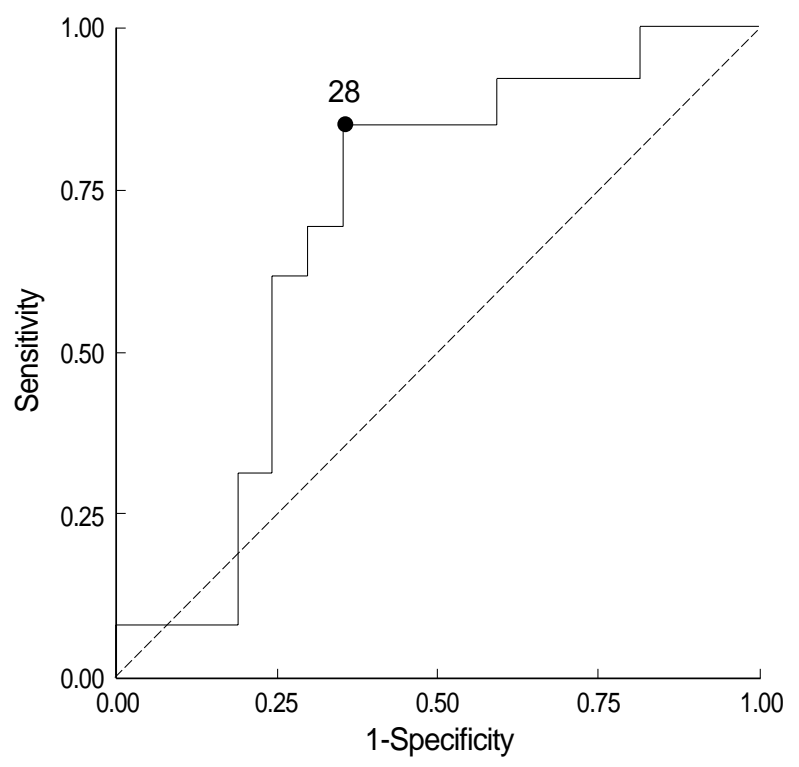

Fig. 1. Receiver operating characteristic curve analysis showed that the curve constructed for cervical length was above the 45degree angle, indicating a significant relationship with the presence of a positive amniotic fluid culture. Number next to solid dot represents cervical length $(\mathrm{mm})$ (area under curve 0.696, SE $0.078, p=0.013)$.

with positive amniotic fluid cultures had significantly lower median gestational age at delivery, shorter median cervical length, and higher median maternal blood CRP concentration, $\mathrm{WBC}$, and $\mathrm{AF} \mathrm{WBC}$ than did those with negative $\mathrm{AF}$ cultures (Table 1).

Fig. 1 displays the ROC curve describing the values of cervical length in the identification of a positive amniotic fluid culture. The curve constructed for sonographically measured cervical length was above the $45^{\circ}$ line, indicating that there was a significant relationship between this variable and the presence of a positive amniotic fluid culture (area under the curve 0.696 ; SE $0.078 ; p=0.013$ ). The best cut-off value in the identification of a positive amniotic fluid culture was 28 mm with a sensitivity of $85 \%$ and a specificity of $65 \%$.

To determine the relative value of cervical length in the identification of microbial invasion of the amniotic cavity, we conducted multiple logistic regression analysis with variables that could be considered risk factors for microbial invasion of the amniotic cavity: cervical length, AF WBC, maternal WBC, and maternal CRP. Of all these independent variables, only the cervical length retained statistical significance in the identification of microbial invasion of the amniotic cavity after other confounding variables were adjusted (Table 2).

\section{DISCUSSION}

The present study demonstrates that cervical length is a sensitive maker of intra-amniotic microbial invasion in PP-
Table 2. Relationship of various independent variables with positive amniotic fluid cultures analyzed by overall logistic regression

\begin{tabular}{lccc}
\hline & Odd ratio & $\begin{array}{c}95 \% \text { confi- } \\
\text { dence interval }\end{array}$ & $\begin{array}{c}p \\
\text { value }\end{array}$ \\
\hline Cervical length $(\mathrm{cm})$ & 0.09 & $0.01-0.95$ & 0.045 \\
Maternal CRP $(\mathrm{mg} / \mathrm{dL})$ & 0.51 & $0.15-1.72$ & $\mathrm{NS}$ \\
Maternal WBC $(\mathrm{celll} / \mu \mathrm{L})$ & 1.82 & $0.79-4.18$ & $\mathrm{NS}$ \\
AF WBC $(\mathrm{cell} s / \mu \mathrm{L})$ & 1.14 & $0.99-1.31$ & 0.071 \\
\hline
\end{tabular}

NS, not significant; CRP, C-reactive protein; WBC, white blood cell count; AF, amniotic fluid.

ROM patients. Moreover, logistic regression analysis, after correcting for potential confounders, showed that cervical length is an independent predictor of intra-amniotic microbial invasion in these patients (odds ratio 0.09, $p=0.045$ ).

A considerable body of evidence supports that microbial invasion of the amniotic cavity elicits maternal and fetal inflammatory responses (17-19). Consequently, women with microbial invasion of the amniotic cavity have a shorter amniocentesis-to-delivery interval and higher frequencies of clinical chorioamnionitis and neonatal complications than those with sterile amniotic fluid $(3,4)$. Moreover, in addition to the established association between fetal exposure to infection and short-term morbidity (20), such exposures have also been associated with long-term sequelae, such as, cerebral palsy $(21,22)$ and chronic lung disease $(23,24)$. Thus, early and accurate detection of microbial invasion of the amniotic cavity remains an important challenge in clinical obstetrics. The gold standard for the diagnosis of microbial invasion of the amniotic cavity remains microbiological culture of amniotic fluid obtained by amniocentesis, but regrettably, culture results may take several days. Accordingly, other more rapid tests have been proposed for the detection of microbial invasion of the amniotic cavity (4, 25-27), but all require amniocentesis and present the risks associated with its invasive nature. On the other hand, maternal blood sampling and analysis can be performed safely in any laboratory, and it has been reported that women with positive AF cultures have statistically significant higher median maternal plasma $\mathrm{C}$-reactive protein concentrations, WBC, and AF WBC than women with negative $\mathrm{AF}$ cultures (3). However, the poor diagnostic performance of maternal plasma $\mathrm{C}$-reactive protein concentration and WBC limit the usefulness of these blood tests for the detection of microbial invasion of the amniotic cavity (3). Although an elevated AF WBC ( $>20$ cells $/ \mu \mathrm{L}$ ) has been reported to have a sensitivity of $84 \%$ for the detection of microbial invasion of the amniotic cavity, this test also requires amniocentesis (3).

In terms of the general management of PPROM patients, cervical dilatation and effacement are estimated visually using a sterile speculum, because a digital examination introduces the risk of infection (28). However, ultrasound can be used to safely assess cervical status in PPROM patients $(12,29)$, 
and cervical length as determined by transvaginal ultrasonography in PPROM patients might predict the interval between admission and delivery $(12,13,29)$. Moreover, recently, it has been reported that cervical length measurement usefully predicts microbial invasion of the amniotic cavity in women with preterm labor and intact membranes $(14,15)$.

In the present study, it was found that cervical length is a sensitive marker (sensitivity $85 \%$; specificity $65 \%$ ) of the presence of intra-amniotic microbial invasion in PPROM patients. Moreover, perhaps the most important finding of the present study is that cervical length $(\leq 28 \mathrm{~mm})$ was found to be a better independent predictor of microbial invasion of the amniotic cavity than amniotic fluid WBC or maternal WBC or CRP. Our findings encourage us to postulate that local inflammatory response induced by intrauterine infection first elicits cervical change and subsequently the recruitment of WBCs to amniotic fluid. This finding has important clinical implications because a cervical length determination may allow clinicians to identify the patients with PPROM most likely to benefit from antibiotics before delivery without the having to perform amniocentesis.

Gomez et al. proposed two reasons why microbial invasion of the amniotic cavity is more common in patients with a short cervix and intact membranes (15). The first is that a short cervix simply represents recruitment of the cervical components of the common terminal pathway of parturition because patients could have microbial invasion of the amniotic cavity as a cause of preterm labor, and the second possibility is that a short cervix may more predispose the individual to an ascending intrauterine infection. However, in cases of PPROM, membrane rupture per se is a risk factor of an ascending intrauterine infection, and therefore, a short cervix can be regarded as a ripened cervix.

Several investigators have consistently shown a relation between cervical length and the risk of perinatal infection. Iams et al. found that a short cervix during pregnancy is an independent risk factor of the subsequent developments of clinical chorioamnionitis and neonatal sepsis (30). Hassan et al. concluded that a short cervix is a clinical manifestation of intra-amniotic infection (14), and Gomez et al. reported that a short cervix in women with preterm labor and intact membranes is a risk factor for microbial invasion of the amniotic cavity (15). Moreover, it has been reported that a short cervix in women with PPROM is a risk factor of imminent delivery $(12,13)$.

In conclusion, the present study shows that transvaginal sonographic measurements of cervical length provide a valuable means for identifying microbial invasion of the amniotic cavity in women with PPROM. Moreover, cervical length was found to be a better predictor for microbial invasion than the AF WBC or maternal blood CRP and WBC determinations.

\section{REFERENCES}

1. Kaltreider DF, Kohl S. Epidemiology of preterm delivery. Clin Obstet Gynecol 1980; 23: 17-31.

2. Gauthier DW, Meyer WJ, Bieniarz A. Correlation of amniotic fluid glucose concentration and intraamniotic infection in patients with preterm labor or premature rupture if membranes. Am J Obstet Gynecol 1991; 165: 11059-10.

3. Yoon BH, Jun JK, Park KH, Syn HC, Gomez R, Romero R. Serum C-reactive protein, white blood cell count, and amniotic fluid white blood cell count in women with preterm premature rupture of membranes. Obstet Gynecol 1996; 88: 1034-40.

4. Romero R, Yoon BH, Mazor M, Gomez R, Gonzalez R, Diamond MP, Baumann P, Araneda H, Kenney JS, Cotton DB. A comparative study of the diagnostic performance of amniotic fluid glucose, white blood cell count, interleukin-6, and gram stain in the detection of microbial invasion in patients with preterm premature rupture of membranes. Am J Obster Gynecol 1993; 169: 839-51.

5. Shim SS, Romero R, Hong JS, Park CW, Jun JK, Kim BI, Yoon BH. Clinical significance of intra-amniotic inflammation in patients with preterm premature rupture of membranes. Am J Obstet Gynecol 2004; 191: 1339-45.

6. Gomez R, Ghezzi F, Romero R, Munoz H, Tolosa JE, Rojas I. Premature labor and intra-amniotic infection. Clinical aspects and role of the cytokines in diagnosis and pathophysiology. Clin Perinatol 1995; 22: 281-342.

7. Jun JK, Yoon BH, Romero R, Kim M, Moon JB, Ki SH, Park JS. Interleukin 6 determinations in cervical fluid have diagnostic and prognostic value in preterm premature rupture of membranes. Am J Obstet Gynecol 2000; 183: 868-73.

8. Shim SS, Romero R, Jun JK, Moon KC, Kim G, Yoon BH. C-reactive protein concentration in vaginal fluid as a marker for intra-amniotic inflammation/infection in preterm premature rupture of membranes. J Matern Fetal Neonatal Med 2005; 18: 417-22.

9. Iams JD Goldenberg RL, Meis PJ, Mercer BM, Moawad Z, Das A, Thom E, McNellis D, Copper RL, Johnson F, Roberts JM. The length of the cervix and the risk of spontaneous premature delivery. $N$ Engl $J$ Med 1996; 334: 567-72.

10. Cook CM, Ellwood DA. The cervix as a predictor of preterm delivery in 'at-risk' women. Ultrasound Obstet Gynecol 2000; 15: 109-13.

11. Hassan SS, Romero R, Berry SM, Dang K, Blackwell SC, Treadwell MC, Wolfe HM. Patients with an ultrasonographic cervical length $<$ or $=15 \mathrm{~mm}$ have nearly a 50\% risk of early spontaneous preterm delivery. Am J Obstet Gynecol 2000; 182: 1458-67.

12. Rizzo G, Capponi A, Angelini E, Vlachopoulou A, Grassi C, Romanini $\mathrm{C}$. The value of transvaginal ultrasonographic examination of the uterine cervix in predicting preterm delivery in patients with preterm premature rupture of membranes. Ultrasound Obstet Gynecol 1998; 11: 23-9.

13. Gire C, Faggianelli P, Nicaise C, Shojai R, Fiori A, Chau C, Boubli L, D'Ercole C. Ultrasonographic evaluation of cervical length in pregnancies complicated by preterm premature rupture of membranes. Ultrasound Obstet Gynecol 2002; 19: 565-9.

14. Hassan S, Romero R, Hendler I, Gomez R, Khalek N, Espinoza J, 
Nien JK, Berry SM, Bujold E, Camacho N, Sorokin Y. A sonographic short cervix as the only clinical manifestation of intra-amniotic infection. J Perinat Med 2006; 34: 13-9.

15. Gomez R, Romero R, Nien JK, Chaiworapongsa T, Medina L, Kim YM, Yoon BH, Carstens M, Espinoza J, Iams JD, Gonzalez R. A short cervix in women with preterm labor and intact membranes: a risk factor for microbial invasion of the amniotic cavity. Am J Obstet Gynecol 2005; 192: 678-89.

16. Yoon BH, Romero R, Kim CJ, Jun JK, Gomez R, Choi JH, Syn HC. Amniotic fluid interleukin-6: a sensitive test for antenatal diagnosis of acute inflammatory lesions of preterm placenta and prediction of perinatal morbidity. Am J Obstet Gynecol 1995; 172: 960-70.

17. Yoon BH, Romero R, Park JS, Kim M, Oh SY, Kim CJ, Jun JK. The relationship among inflammatory lesions of the umbilical cord (funisitis), umbilical cord plasma interleukin 6 concentration, amniotic fluid infection, and neonatal sepsis. Am J Obstet Gynecol 2000; 183: 1124-9.

18. Pacora P, Chaiworapongsa T, Maymon E, Kim YM, Gomez R, Yoon BH, Ghezzi F, Berry SM, Qureshi F, Jacques SM, Kim JC, Kadar N, Romero R. Funisitis and chorionic vasculitis: the histological counterpart of the fetal inflammatory response syndrome. J Matern Fetal Meonatal Med 2002; 11: 18-25.

19. Salafia CM, Popek EJ. Placenta. In: Damjanov I, Linder J (eds) Anderson's pathology. St. Louis: Mosby-Year Book Inc; 1996; 2310-53.

20. Gomes R, Romero R, Ghezzi F, Yoon BH, Mazor M, Berry SM. The fetal inflammatory response syndrome. Am J Obstet Gynecol 1998; 179: 174-202.

21. Yoon BH, Jun JK, Romero R, Park KH, Gomez R, Choi JH, Kim IO. Amniotic fluid inflammatory cytokines (interleukin-6, interleukin-lbeta, and tumor necrosis factor-alpha), neonatal brain white matter lesions, and cerebral palsy. Am J Obstet Gynecol 1997; 177: 19-26.

22. Yoon BH, Romero R, Park JS, Kim CJ, Kim SH, Choi JH, Han TR.
Fetal exposure to an intra-amniotic inflammation and the development of cerebral palsy at the age of three years. Am J Obstet Gynecol 2000; 182: 675-81.

23. Ghezzi F, Gomez R, Romero R, Yoon BH, Edwin SS, David C, Janisse J, Mazor M. Elevated interleukin-8 concentrations in amniotic fluid of mothers whose neonates subsequently develop bronchopulmonary dysplasia. Eur J Obstet Gunecol Reprod Biol 1998; 78: 5-10.

24. Yoon BH, Romero R, Kim KS, Park JS, Ki SH, Kim BI, Jun JK. A systemic fetal inflammatory response and the development of bronchopulmonary dysplasia. Am J Obstet Gynecol 1999; 181: 773-9.

25. Romero R, Emamian M, Quintero R, Wan M, Hobbins JC, Mazor $\mathrm{M}$, Edberg $\mathrm{S}$. The value and limitations of gram stain examination in diagnosis of intraamniotic infection. Am J Obatet Gynecol 1988; 159: 144-9.

26. Romero R, Emamian M, Wan M, Yarkoni S, McCormack W, Mazor $\mathrm{M}$, Hobbins JC. The value of the leukocyte esterase test in diagnosing intra-amniotic infection. Am J Perinatol 1988; 5: 64-9.

27. Yoon BH, Romero R, Kim CJ, Jun JK, Gomez R, Choi JH, Syn HC. Amniotic fluid interleukin-6: a sensitive test for antenatal diagnosis of acute inflammatory lesions of preterm placenta and prediction of perinatal morbidity. Am J Obstet Gynecol 1995; 172: 960-70.

28. Schutte MF, Treffers PE, Kloosterman GJ, Soepatmi S. Management of premature rupture of membranes: the risk of vaginal examination to the infant. Am J Obstet Gynecol 1983; 146: 395-400.

29. Carlan SJ, Richmond LB, O'Brien WF. Randomized trial of endovaginal ultrasound in preterm premature rupture of membranes. Obstet Gynecol 1997; 89: 458-61.

30. Iams JD, for the NICHD MFMU Network. Maternal Fetal Medicine Unit Network, BM. The Preterm Prediction Study: cervical length and perinatal infection. Am J Obstet Gynecol 1997; 176: S6. 